\title{
Rifabutin-Cobicistat Drug Interaction Resulting in Severe Bilateral Panuveitis
}

\author{
Christopher B. Toomey Jeffrey Lee Doran B. Spencer
}

Shiley Eye Institute, Viterbi Family Department of Ophthalmology, University of California San Diego, La Jolla, CA, USA

\section{Keywords}

Rifabutin · Retina $\cdot$ Drug toxicity $\cdot$ Uveitis $\cdot$ Human immunodeficiency virus

\begin{abstract}
We report a novel case of severe bilateral panuveitis with hypopyon secondary to rifabutin and cobicistat drug interaction in the setting of human immunodeficiency virus (HIV) infection and latent tuberculosis (TB). A 63-year-old woman presented with bilateral conjunctival injection and decreasing vision of 5 days' duration. She had a history of well-controlled HIV infection, latent $\mathrm{TB}$, and non-alcoholic steatohepatitis for which she was inadvertently being treated, due to a pharmacy error, concurrently with the anti-TB medicine rifabutin and the highly active antiretroviral therapy combination Genvoya ${ }^{\circledR}$ (elvitegravir $150 \mathrm{mg}$ - cobicistat $150 \mathrm{mg}$ emtricitabine $200 \mathrm{mg}$ - tenofovir alafenamide $10 \mathrm{mg}$ ). Ocular examination was significant for bilateral panuveitis with hypopyon. Blood, cerebrospinal fluid, and vitreous analysis were negative for infectious or rheumatologic abnormalities. Rifabutin was discontinued and the patient was treated with intravenous followed by oral steroids as an outpatient with eventual resolution of symptoms. This unique case of rifabutin-cobicistat drug interaction highlights the association between rifabutin drug levels and ocular inflammation and expands the potential presentation of rifabutin-associated uveitis to include bilateral panuveitis with hypopyon.
\end{abstract}




\section{Case Reports in Ophthalmology}

\section{Introduction}

Uveitis in the setting of infection with the human immunodeficiency virus (HIV) represents a potentially serious, vision-threatening clinical conundrum. The uveitis may stem from a multitude of etiologies, specifically infectious and non-infectious, that require opposing treatment regimens. Infectious causes of uveitis in an HIV patient span the microbial spectrum and may originate from bacterial, viral, fungal, or parasitic microorganisms. The evaluation and diagnostic work-up is typically dictated by the degree of immunosuppression, as represented by the CD4+ T cell count, and clinical presentation. Non-infectious etiologies may include routine autoimmune uveitis conditions, but malignant "masquerade" syndromes must also be considered, as HIV patients irrespective of immunosuppressive status incur a 10-fold greater likelihood of malignancy than the normal population.

Approximately 36.9 million people worldwide were estimated to be infected with HIV in 2017, and one-quarter of the world population is infected with tuberculosis (TB). Co-infection with HIV and TB, which occurs in 1.2 million people, is a serious public health concern due to the increased risk of reactivation of latent TB and accounts for around one-third of AIDS-related deaths. For this reason, co-infected patients are typically treated concomitantly with highly active antiretroviral therapy and anti-TB treatment. The United Nation's Stop TB Partnership's Global Plan describes the goal of co-treatment of all HIV-positive TB patients in the coming years. We describe here a unique case involving a previously unreported uveitis presentation due to a specific drug-drug interaction from concomitant highly active antiretroviral therapy and anti-TB therapy. Our goal is to educate the ophthalmologic as well as the broader medical community about a possibly increasingly common clinical scenario and to expand the known potential presentation of rifabutin-associated uveitis to include severe bilateral panuveitis with hypopyon.

\section{Case Report}

A 63-year-old female was referred from an outside hospital due to bilateral ocular redness and decreasing vision of 5 days' duration with only mild ocular irritation. Review of systems revealed 3 weeks of progressively worsening arthralgias, subjective fevers, and headache. The patient had a history of well-controlled HIV infection although without recent blood work (CD4 count 630 from 6 months prior), latent TB status post treatment, and cirrhosis secondary to non-alcoholic steatohepatitis, all managed by an outside hospital. The patient had been treated for latent TB with rifabutin $300 \mathrm{mg}$ daily for a 4-month treatment course 9 months prior. Rifabutin was chosen for the treatment instead of isoniazid due to the nonalcoholic steatohepatitis. However, due to pharmacy error, the patient was restarted on rifabutin $300 \mathrm{mg}$ daily for 3 months prior to admission while concurrently on Genvoya ${ }^{\circledR}$ (elvitegravir $150 \mathrm{mg}$ - cobicistat $150 \mathrm{mg}$ - emtricitabine $200 \mathrm{mg}$ - tenofovir alafenamide $10 \mathrm{mg}$ ) for HIV management which she had started 5 months prior to admission.

Ocular examination revealed a best corrected visual acuity of 20/70 in the right eye and count fingers at 4 feet in the left eye. Intraocular pressure was within normal limits in both eyes. Pupils were reactive in both eyes without relative afferent pupillary defect, and extraocular movements showed no limitations. Anterior segment examination was significant for 2+ injection of the conjunctiva in both eyes and a $<1 \mathrm{~mm}$ hypopyon with $4+$ anterior chamber cell and flare with fibrin attached to the anterior lens capsule in both eyes. Posterior examination 


\section{Case Reports in Ophthalmology}

Case Rep Ophthalmol 2020;11:156-160

DOI: $10.1159 / 000506181$

(c) 2020 The Author(s). Published by S. Karger AG, Basel www.karger.com/cop

Toomey et al.: Rifabutin-Associated Bilateral Panuveitis

showed 4+ anterior and posterior vitritis with only a limited view of the inferior retina showing several opaque white vitreous opacities in the right eye.

Due to the uncertain immunosuppressive state on presentation as well as systemic complaints, an extensive infectious work-up was performed in an inpatient setting in concert with the Infectious Diseases Service. Serum syphilis enzyme immunoantibody, rapid plasma reagin, Borrelia burgdorferi antibody, CMV IgG/IgM, varicella IgG, and HSV type 1/2 IgM were negative. Quantiferon-TB was positive and chest CT showed no evidence of intrathoracic TB. Serum angiotensin-converting enzyme, C-reactive protein, and erythrocyte sedimentation rate were within normal limits. CT head and lumbar puncture showed no evidence of neurological involvement. A vitreous sample was obtained from the left eye. Gram stain showed polymorphonucleated white blood cells without organisms and cultures showed no growth. The encephalitis panel polymerase chain reaction was administered to the vitreous tap sample and was negative; this included: enterovirus, HSV type 1/2, herpesvirus 6, parechovirus, varicella zoster, Cryptococcus neoformans/gattii, Escherichia coli K1, Haemophilus influenzae, Listeria monocytogenes, Neisseria meningitidis, Streptococcus agalactiae, Streptococcus pneumoniae, and cytomegalovirus. On day 1 of hospitalization, vision had worsened to count fingers and hand motion at near without correction (sc) although without development of significant ocular discomfort.

Due to the high suspicion for a non-infectious etiology, the patient was initially treated with cycloplegia and topical prednisolone with discontinuation of rifabutin, and vision and inflammation improved after 3 days, without other treatment; visual acuity on hospital day 3 was sc 20/400 and count fingers at near with cycloplegia. After infectious work-up had been negative, the patient was started on intravenous methylprednisolone $250 \mathrm{mg}$ q.i.d. for 1 day and transitioned to oral prednisone at discharge, $60 \mathrm{mg}$ daily with weekly taper. At 10-day follow-up the patient's arthralgias had resolved, her visual acuity was cc 20/200 and 20/400 at distance with resolving anterior segment inflammation and persistent vitritis (Fig. 1a, b). At 3-month follow-up her visual acuity was cc 20/40 and 20/40 at distance with mild residual vitritis (Fig. 1c, d).

\section{Discussion}

Rifabutin-associated uveitis has been recognized as a dosage-dependent side effect, typically resulting in anterior uveitis [1-4]. Our case appears to be the first reported incidence of severe bilateral panuveitis with hypopyon due to rifabutin toxicity. The severity of inflammation seen in this case is not typically associated with rifabutin-associated uveitis. We propose that this unusual manifestation occurred due to an adverse drug-drug interaction from the concurrent rifabutin and cobicistat usage along with baseline liver pathology, resulting in exceptionally severe ocular inflammation from rifabutin.

Rifabutin is metabolized by the cytochrome enzyme CYP3A [5]. Cobicistat, a CYP3A inhibitor, has been shown to increase 25-0-rifabutin area under the curve and minimal concentration levels (625 and 494\%, respectively) compared to rifabutin alone [6, 7]. Several case reports have shown an association between rifabutin and uveitis usually in the setting of Mycobacterium infection [3, 8, 9] or prophylaxis for Mycobacterium infection [2, 10]. However, these reports typically report anterior uveitis, and the etiology is complicated by concurrent systemic infection or immunosuppression. Here we report a case where an inadvertent drugdrug interaction led to severe rifabutin-associated panuveitis in a well-controlled HIV patient without active mycobacterial disease, but with concomitant cirrhosis and treatment with a 
known CYP inhibitor. This further highlights the link between serum rifabutin levels and ocular inflammation. Given the increasingly frequent co-infection with HIV and mycobacteria, the United Nations' goals of increasing co-treatment for these infections in upcoming years, and the severity of the ocular inflammation noted in this case during concurrent treatment, we believe that this case warrants significant attention from HIV and uveitis specialists.

\section{Statement of Ethics}

This research complied with the guidelines for human studies and was conducted ethically in accordance with the World Medical Association Declaration of Helsinki.

\section{Disclosure Statement}

All authors have no conflicts of interest to disclose.

\section{Funding Sources}

There was no funding source for this study.

\section{Author Contributions}

All authors contributed to clinical care, data gathering, preparation, and editing of the manuscript.

\section{References}

1 Becker K, Schimkat M, Jablonowski H, Häussinger D. Anterior uveitis associated with rifabutin medication in AIDS patients. Infection. 1996 Jan-Feb;24(1):34-6.

2 Nichols CW. Mycobacterium avium complex infection, rifabutin, and uveitis - is there a connection? Clin Infect Dis. 1996 Apr;22 Suppl 1:S43-7; discussion S47-9.

3 Jetton J, Gaviria JG, Patterson TF, Johnson DA. Rifabutin-associated uveitis in an immunocompetent individual with Mycobacterium simiae. Can J Ophthalmol. 2009 Aug;44(4):468-9.

4 Gioulekas J, Hall A. Uveitis associated with rifabutin therapy. Aust N Z J Ophthalmol. 1995 Nov;23(4):319-21.

5 Burman WJ, Gallicano K, Peloquin C. Comparative pharmacokinetics and pharmacodynamics of the rifamycin antibacterials. Clin Pharmacokinet. 2001;40(5):327-41.

6 STRIBILD package insert. Gilead Sciences, Inc.; 2012. Available from: www.gilead.com/ /media/Files/pdfs/ medicines/hiv/stribild/stribild_pi.pdf.

7 Guidelines for the use of antiretroviral agents in pediatric HIV infection. Department of Health and Human Services; 2013. Available from: https://aidsinfo.nih.gov/guidelines/html/2/pediatric-arv/45/whats-newin-the-guidelines.

8 Wang HH, Chung YM, Lin YC, Hu HC, Wong WW. Rifabutin-induced hypopyon uveitis in patients with acquired immunodeficiency syndrome infected with Mycobacterium avium complex. J Chin Med Assoc. 2007 Mar;70(3):136-9.

9 Fineman MS, Vander J, Regillo CD, Fineman SW, Brown GC. Hypopyon uveitis in immunocompetent patients treated for Mycobacterium avium complex pulmonary infection with rifabutin. Retina. 2001;21(5):531-3.

10 Rifai A, Peyman GA, Daun M, Wafapoor H. Rifabutin-associated uveitis during prophylaxis for Mycobacterium avium complex infection. Arch Ophthalmol. 1995 Jun;113(6):707. 


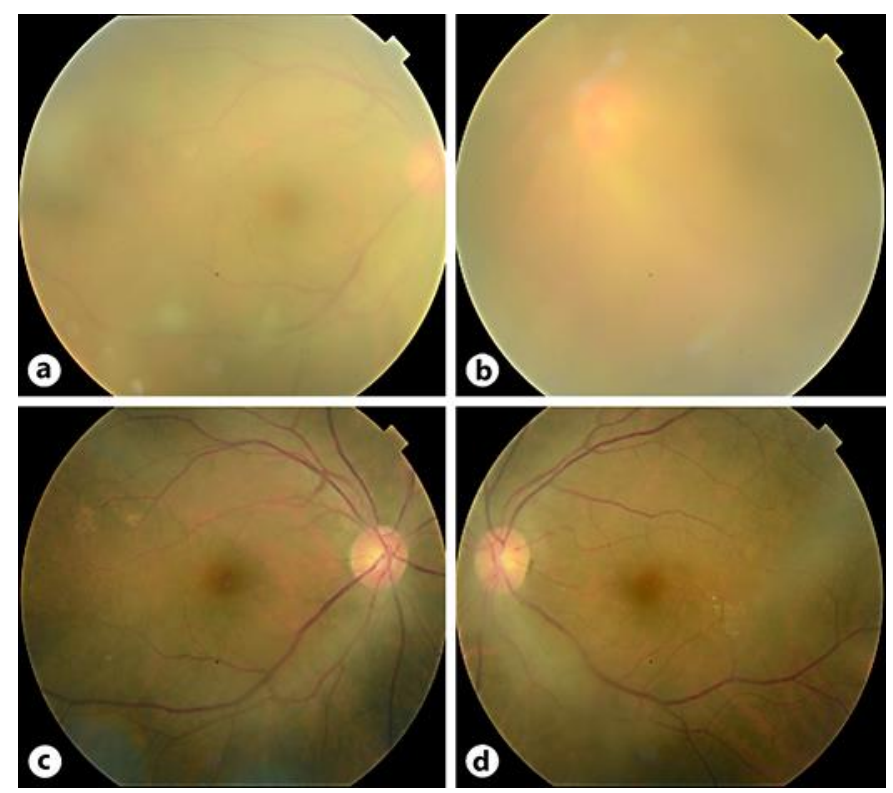

Fig. 1. a, b Fundus photographs of the right (a) and left (b) eye showing vitritis with visible aggregates of inflammatory cells inferiorly in the left eye. c, $\mathbf{d}$ Fundus photographs at 3-month follow-up of the right (c) and left (d) eye showing resolution of the vitritis. 